\title{
NUEVAS TECNOLOGÍAS DE LA INFORMACIÓN Y LA COMUNICACIÓN, (NTIC): DIAGNÓSTICO EN LAS DIFERENTES CARRERAS QUE SE ESTUDIAN EN LA UPAP, FILIAL PILAR, PARAGUAY NEW INFORMATION AND COMMUNICATION TECHNOLOGIES (NTIC): DIAGNOSIS IN THE DIFFERENT CAREERS STUDIED AT UPAP, SUBSIDIARY PILAR, PARAGUAY
}

\author{
Lorena del Rocío Silvera Medina ${ }^{1}$ \\ Arnoldo Eduardo Álvarez López ${ }^{2}$ \\ 1 Universidad Politécnica y Artística de Paraguay, UPAP \\ lorenasilvera@hotmail.es \\ (iD) de ORCID: https://orcid.org/0000-0002-1269-659X \\ 2 Universidad Politécnica y Artística del Paraguay \\ arnoldoeduardo56@gmail.com \\ (iD) de ORCID: https://orcid.org/0000-0002-9426-0297 \\ 1 Licenciada. Directora de la Filial, Universidad Politécnica y Artística de Paraguay, UPAP, Pilar. \\ 2 Arquitecto. Doctor en Ciencias. Docente e Investigador. Coordinador de Extensión e Investigación. \\ Universidad Politécnica y Artística de Paraguay, UPAP, Pilar - Paraguay.
}

Fuente de financiamiento: Autofinanciado

Declaración de conflictos de interés: Los autores afirman no poseer conflictos de interés.

\begin{abstract}
Resumen
El uso de Nuevas Tecnologías de la Información y la Comunicación (NTIC), en la enseñanza superior en Paraguay y particularmente en la Universidad Politécnica y Artística de Paraguay (UPAP), cobra mayor relevancia en la situación actual del país y del mundo envuelto en una pandemia, para no detener el curso docente. En tal sentido, el trabajo tiene como objetivo diagnosticar el uso de las NTIC en las carreras que se estudian en la UPAP, filial Pilar. Constituye una investigación deductiva, interpretativa y descriptiva; puede ser un estudio piloto y servir como antecedente, para su generalización en otras filiales del país, para poder trazar estrategias educativas, pedagógicas, conocer las relaciones alumnos - computadoras en cada centro, en cada hogar, saber de las conectividades necesarias para implementar estas herramientas y que se hagan más eficientes los diferentes cursos, logrando mayor eficacia en el proceso enseñanza aprendizaje. Se ilustran los análisis y resultados, y se arriba a la conclusión de que se necesita profundizar en la utilización de las NTIC, pues hay poco conocimiento y no existen suficientes proyectos para elevar estas herramientas al plano necesario, y además existe un déficit de máquinas por estudiante y poca conectividad, entre otros aspectos.
\end{abstract}




\begin{abstract}
The use of New Information and Communication Technologies (NTIC) in higher education in Paraguay, and particularly at the Polytechnic and Artistic University of Paraguay, (UPAP), becomes more relevant in the current situation of the country and of the world involved in a pandemic, so as not to stop the teaching course. In this sense, the objective of the work is to diagnose the use of NTIC in the careers studied at UPAP, Subsidiary Pilar. It constitutes a deductive, interpretive and descriptive research, it can be a pilot study and serve as an antecedent, for its generalization in other subsidiaries of the country, to be able to trace educational, pedagogical strategies, know the student-computer relationships in each center, in each home, know the connectivity necessary to implement these tools and make the different courses more efficient, making the teaching-learning process more effective. The analyzes and results are illustrated, and the conclusion is reached, that it is necessary to deepen the use of NICTs, there is little knowledge, there are not enough projects to raise these tools to the necessary level, there is a deficit of machines per student and little connectivity in other aspects.
\end{abstract}

Key words: Diagnosis, new information and communication technologies, teachinglearning process. 


\section{Introducción}

Las Nuevas Tecnologías de la Información y Comunicación (NTIC), alcanzan un nivel de prestaciones cada día más alto, así como mayor calidad técnica, innovación y sofisticación. En tal sentido, el proceso de enseñanza aprendizaje y la formación académica en todos los niveles y sobre todo en el ámbito universitario, no son ajenos a la necesidad de su utilización, de aprovechar las potencialidades y recursos que ofrecen y que ponen a disposición de la academia. y de la docencia universitaria.

Según autores como Ávila (2003) y Domínguez (2016), en América Latina y el Caribe, el presente y futuro del proceso enseñanza-aprendizaje, con la utilización de las NTIC en la educación superior, pasa por "la producción de conocimiento que, si bien ha demostrado avances, presenta todavía graves debilidades con una baja incorporación de internet, NTIC y sus derivados". Esto lleva a plantear que se manifiestan problemas no resueltos para la incorporación de estas nuevas tecnologías educativas, y que se necesita de docentes e investigadores que encuentren soluciones creativas a antiguos y nuevos problemas, en este tema.

En el contexto del Paraguay, existen docentes y futuros egresados que en breve tiempo serán docentes universitarios, inconformes con las propuestas formativas relacionadas con NTIC, orientadas a la práctica académica en la educación superior. (Agencia Nacional de Evaluación y Acreditación de la Educación Superior ANEAES, 2013; Argüello, 2018).

Esto se contradice con las experiencias actuales en el contexto de la pandemia de COVID-19, que ha llevado al uso de las NTIC para no parar el proceso enseñanza-aprendizaje en el país y no atrasar los cursos de las diferentes carreras, buscando alternativas para ello. Y es allí donde las NTIC son herramientas fundamentales.

Por otra parte, si esto es una realidad, la otra cara es que muchos profesores universitarios no se sienten capaces de utilizarlas para desarrollar competencias profesionales y académicas, además de las propias necesidades del hombre en la comunicación.

Otro argumento importante es que, en los procesos de autoevaluación de carreras universitarias que realiza la Agencia Nacional de Evaluación y Acreditación de la Educación Superior (ANEAES) a nivel nacional, cada vez son más frecuentes en sus observaciones e incluso en las quejas de los propios estudiantes, la poca o escasa utilización de NTIC en las asignaturas y plataformas educativas.

Se señala, en estas circunstancias, que la docencia universitaria en Paraguay pasa por un interesante proceso de institucionalización, perfeccionamiento y profesionalización, motivado principalmente por la propia ANEAES en sus procesos de acreditación y orientación, al evaluar la calidad de las carreras universitarias. Esta institución fomenta que los claustros académicos se capaciten de forma permanente, en función de elevar sus conocimientos y de cumplir con eficiencia y eficacia sus funciones académicas. El uso de NTIC se encuentra entre los indicadores de calidad, según sus propias fuentes (ANEAES, 2016; Valenzuela, 2017).

Este trabajo intenta aportar en esta dirección y proponer acciones para atender las necesidades de formación, de estudiantes de diferentes carreras universitarias que estudian en la UPAP, filial de Pilar, en referencia al aprovechamiento de NTIC.

Se pretende cumplir con el Consejo Nacional de Ciencia y Tecnología (CONACYT) en sus programas y que, en opinión de los autores, se haga manifiesta la necesidad y oportunidad de investigar sobre el uso de las NTIC, con miras a mejorar la impartición de conocimientos, optimizar tiempo, recursos y esfuerzos en el proceso enseñanza-aprendizaje de cada carrera. (CONACYT, s.f.).

El propósito de este trabajo es diagnosticar el uso de las NTIC en las diferentes carreras habilitadas y que se estudian en la UPAP, filial Pilar en Paraguay, en el contexto del proceso enseñanza - aprendizaje, en el año 2020 en momentos de la Pandemia COVID 19, como institución de la educación superior.

\section{NUEVAS TECNOLOGÍAS DE LA INFORMACIÓN Y LA COMUNICACIÓN, (NTIC): DIAGNÓSTICO EN LAS DIFERENTES CARRERAS QUE SE ESTUDIAN EN LA UPAP, FILIAL PILAR, PARAGUAY NEW INFORMATION AND COMMUNICATION TECHNOLOGIES (NTIC): DIAGNOSIS IN THE DIFFERENT CAREERS STUDIED AT UPAP, SUBSIDIARY PILAR, PARAGUAY}




\section{Metodología}

La UPAP filial Pilar está compuesta por un edificio que reúne todos los requisitos para una instalación de educación superior. Esta institución ha mantenido, en promedio, matrículas de 70 estudiantes cursando diferentes carreras que se exponen a continuación, así como el plantel docente de aquellas que fueron estudiadas para realizar el diagnóstico de las NTIC.

\section{Imagen 1.}

Vista aérea de ubicación de la filial UPAP en Pilar. Fuente: Google Maps.

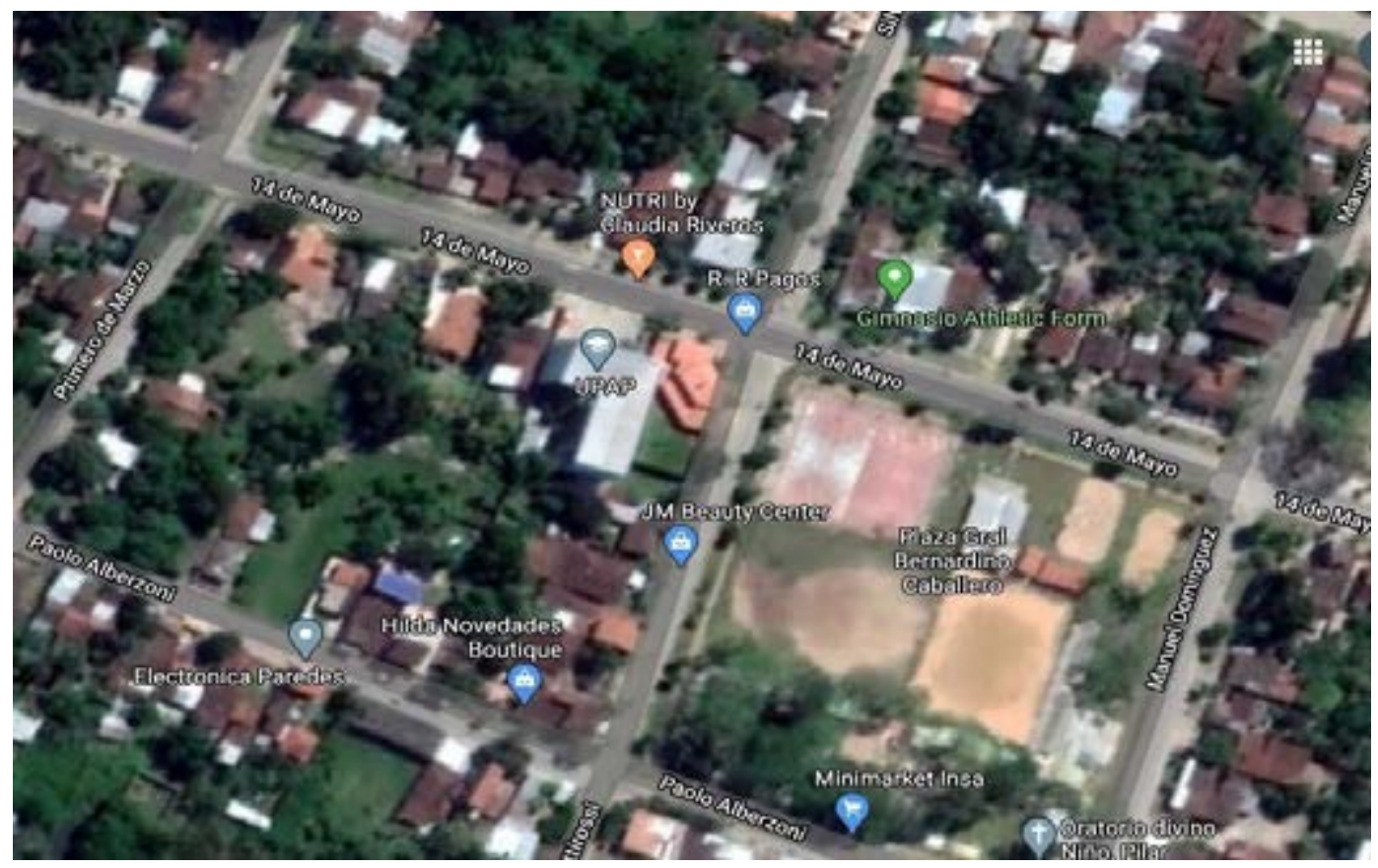

En la UPAP, filial Pilar existen 16 carreras habilitadas en este año 2020. En este apartado se describe en general que la población o universo son las 13 carreras que se estudian y que cuentan con estudiantes en la UPAP. Para la muestra se seleccionaron 12 carreras, en función de posibles docentes para contestar la encuesta-entrevista y de que los resultados fueran representativos. (UPAP, s.f.). Las carreras sometidas a diagnóstico fueron:

Administración de Empresas

Arquitectura

Comercio Exterior

Comunicación Bilingüe Castellano

Diseño y Comunicación Gráfica

Educación Artística

Educación Inicial

Ingeniería Eléctrica

Kinesiología y Fisioterapia

Nutrición

Obstetricia

Psicología

NUEVAS TECNOLOGÍAS DE LA INFORMACIÓN Y LA COMUNICACIÓN, (NTIC): DIAGNÓSTICO EN LAS DIFERENTES CARRERAS QUE SE ESTUDIAN EN LA UPAP, FILIAL PILAR, PARAGUAY NEW INFORMATION AND COMMUNICATION TECHNOLOGIES (NTIC): DIAGNOSIS IN THE DIFFERENT CAREERS STUDIED AT UPAP, SUBSIDIARY PILAR, PARAGUAY 
En relación con el equipamiento de la UPAP en una mirada a las NTIC, se puede comentar que es mínimo y que no cumple las expectativas para el uso eficiente de las mismas. El laboratorio es pequeño, la conectividad es baja, la relación alumno-máquina no es la necesaria, solo lo es si se consideran equipamientos personales de teléfono y computadoras. Por otra parte, faltan terminales y equipos y mobiliarios para un buen funcionamiento. Esto se puede apreciar en las figuras que muestran imágenes del edificio y su equipamiento.

\section{Imagen 2.}

Edificio principal UPAP. Fuente: Autores.
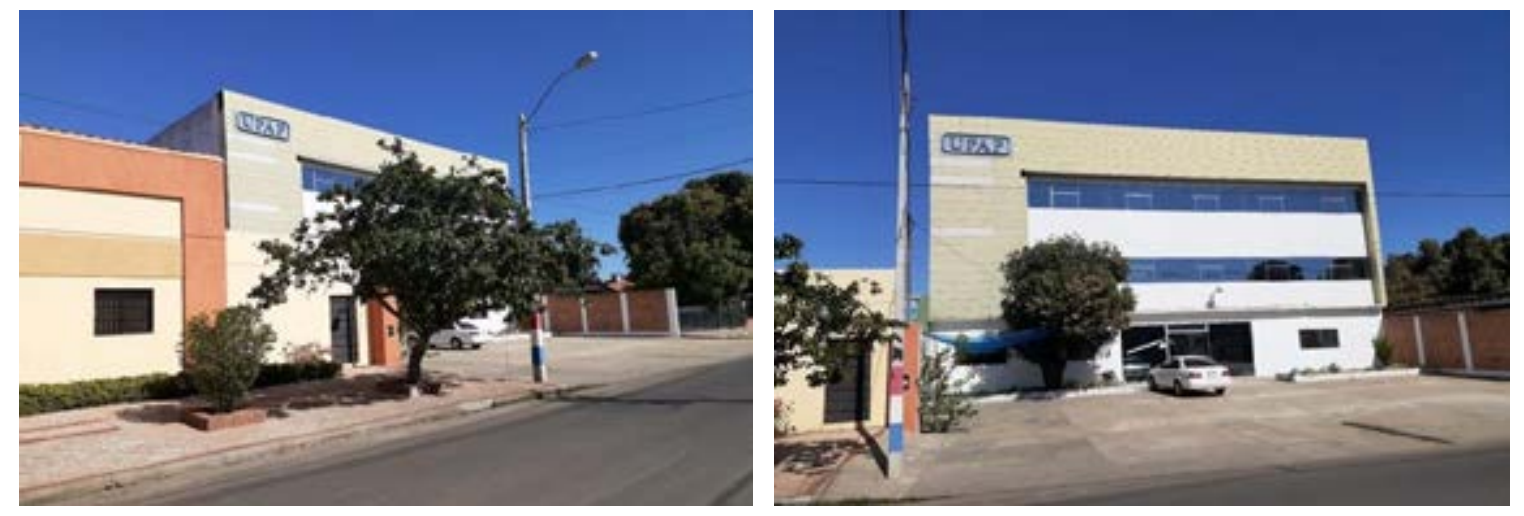

Imagen 3.

Secretaría General UPAP filial Pilar. Fuente: Autores.
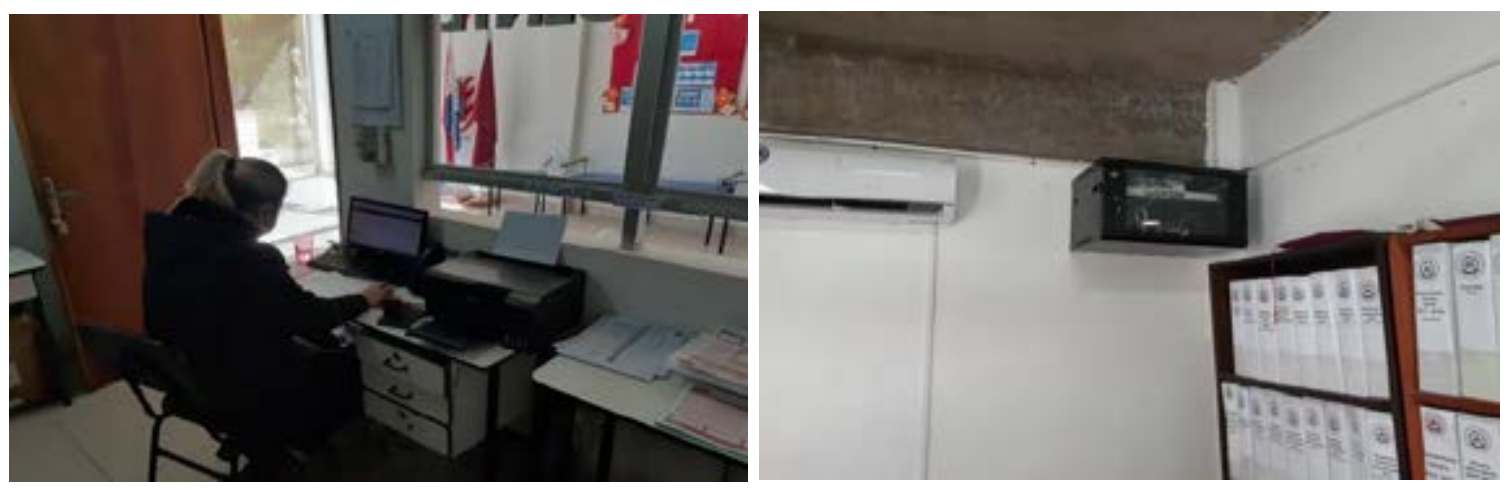

NUEVAS TECNOLOGÍAS DE LA INFORMACIÓN Y LA COMUNICACIÓN, (NTIC): DIAGNÓSTICO EN LAS DIFERENTES CARRERAS QUE SE ESTUDIAN EN LA UPAP, FILIAL PILAR, PARAGUAY NEW INFORMATION AND COMMUNICATION TECHNOLOGIES (NTIC): DIAGNOSIS IN THE DIFFERENT CAREERS STUDIED AT UPAP, SUBSIDIARY PILAR, PARAGUAY 


\section{Imagen 4}

Edificio Docente UPAP filial Pilar. Bloque de Aulas Talleres. Fuente: Autores.
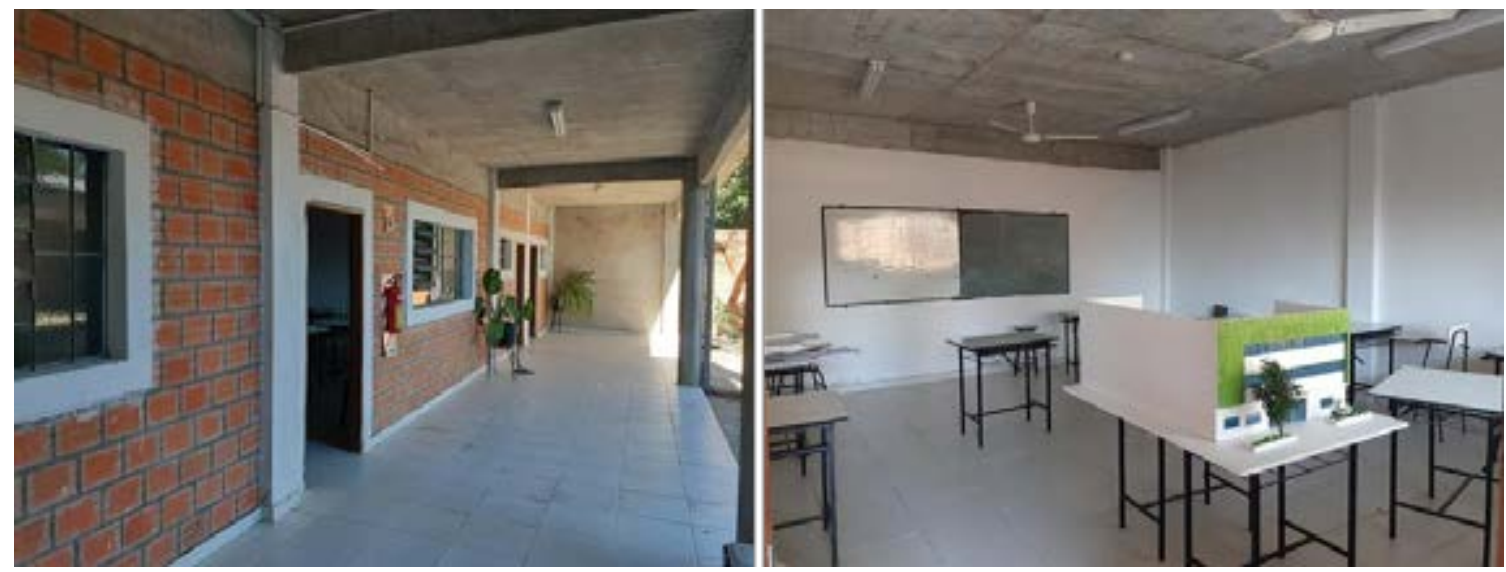

Imagen 5.

Edificio Docente UPAP filial Pilar. Biblioteca - Laboratorio de Computación. Fuente: Autores.
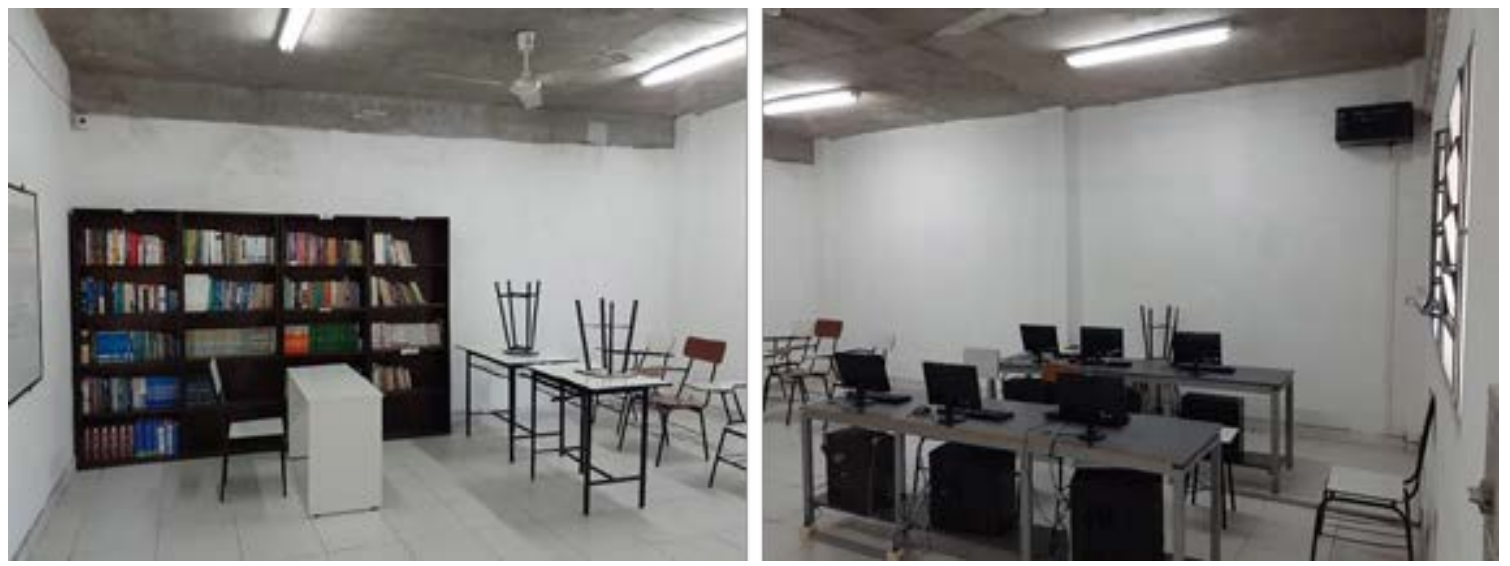

El estudio fue exploratorio, descriptivo e interpretativo, por análisis coyuntural del año 2020, en el contexto de clases digitales por la pandemia del COVID-19. Se analizaron las asignaturas que están aprobadas, pero, sobre todo, las que se imparten por la presencia de estudiantes en la UPAP filial Pilar. Se valoraron las experiencias de docentes según encuestas-entrevistas realizadas, se utilizaron la inducción-deducción como herramientas. Además, se hizo un análisis histórico lógico del tema y se revisaron fuentes bibliográficas o documentales (Baptista et. al., 2014; Zárate et al., 2017). Toda investigación va de lo general a lo particular y en este caso se parte de estudiar las características de cada carrera, su malla curricular y las experiencias y realidades del uso de las NITC.

El desarrollo del estudio se dividió en tres fases o etapas:

- Análisis. Recopilación y análisis de información.

- Síntesis. Encuesta y entrevistas.

- Resultados. Valoración y discusión de los resultados de las entrevistas y encuestas.

NUEVAS TECNOLOGÍAS DE LA INFORMACIÓN Y LA COMUNICACIÓN, (NTIC): DIAGNÓSTICO EN LAS DIFERENTES CARRERAS QUE SE ESTUDIAN EN LA UPAP, FILIAL PILAR, PARAGUAY NEW INFORMATION AND COMMUNICATION TECHNOLOGIES (NTIC): DIAGNOSIS IN THE DIFFERENT CAREERS STUDIED AT UPAP, SUBSIDIARY PILAR, PARAGUAY 


\section{Conclusiones}

El enfoque de este trabajo puede definirse con un alcance exploratorio-descriptivo. En cuanto al diseño metodológico, en relación a las carreras, se generaron y validaron los ítems de una encuesta online. Al término, se dio una noción de la pertinencia de la propuesta educativa para atender las necesidades formativas sobre NTIC, en las diferentes carreras de la UPAP, para docentes y futuros docentes universitarios.

Asimismo, se utilizó la técnica de la entrevista para conocer el parecer experto de los informantes clave, es decir, reconocidos docentes referentes en el ámbito de las NTIC en la UPAP y sus carreras.

\section{Resultados y discusión}

Después de los datos generales de las carreras analizadas y de referencias para la aplicación de la encuesta, se estudiaron las mallas curriculares de las seleccionadas y en total se vieron implicadas 25 asignaturas que utilizan NTIC en el semestre académico, en momentos de la pandemia por COVID-19. Estas carreras tienen, según los resultados de las encuestas, 52 estudiantes. En tres de las encuestas se consigna el desconocimiento del número de estudiantes matriculados en la carrera. No obstante, según averiguaciones de los autores del trabajo, en el presente semestre hay alrededor de 70 estudiantes, pero la cantidad fluctúa mucho por problemas de pago, como consecuencia económica de la pandemia.

Otro resultado revela que el $100 \%$ de los encuestados manifiestan que la UPAP filial Pilar cuenta con un laboratorio de computación, ubicado en el mismo espacio de la biblioteca, que tiene 6 computadoras, lo que da una relación alumno computadora de: 0,09 computadoras por estudiante (no se llega a una computadora por estudiante), una tasa extremadamente baja para una institución de educación superior.

Esta situación se palía de alguna manera con las computadoras individuales de propiedad de cada estudiante, que permiten alcanzar una relación de 0,68 \%. Además, esto imposibilita las clases a través de la plataforma CANVAS que es la que se utiliza en la UPAP como herramienta de las NTIC. Por otra parte, el $100 \%$ de los estudiantes tienen teléfonos con conectividad, pero no les es posible, en opinión de ellos mismos, trabajar desde la casa en la plataforma CANVAS. Por lo tanto, se deduce que esta herramienta no es eficiente para este tipo de clases a distancia por medio de los teléfonos. Se establecen tres grupos de edades, predomina el grupo mayores de 25 años, muy cerca el de 21 a 25 años y el grupo menos representado es el menor a 20 años.

Figura 1.

Relación de edades de los estudiantes que cursan carreras en la UPAP filial Pilar. Año 2020.

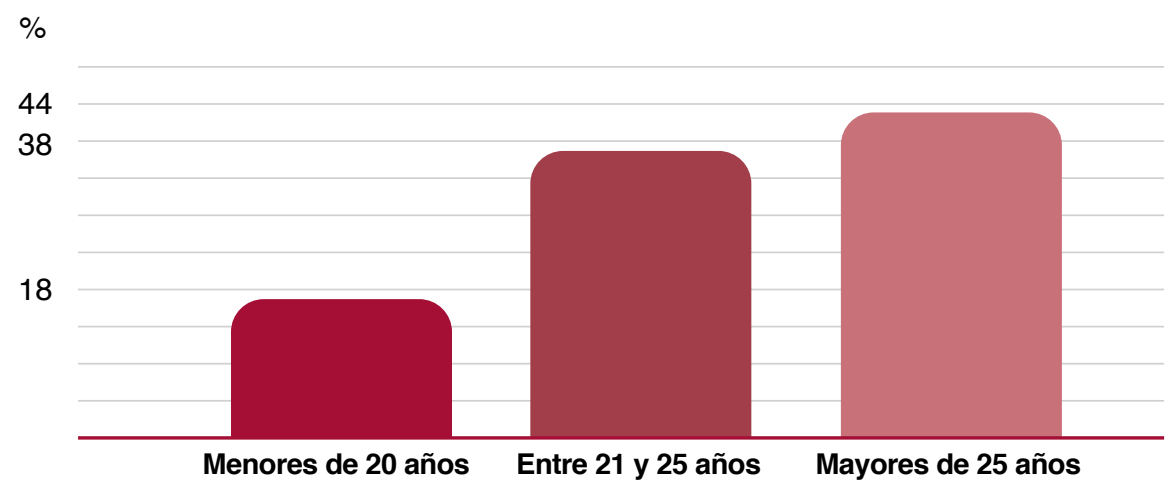

Fuente: Autores.

\section{NUEVAS TECNOLOGÍAS DE LA INFORMACIÓN Y LA COMUNICACIÓN, (NTIC): DIAGNÓSTICO EN LAS DIFERENTES CARRERAS QUE SE ESTUDIAN EN LA UPAP, FILIAL PILAR, PARAGUAY NEW INFORMATION AND COMMUNICATION TECHNOLOGIES (NTIC): DIAGNOSIS IN THE DIFFERENT CAREERS STUDIED AT UPAP, SUBSIDIARY PILAR, PARAGUAY}


El $100 \%$ de los encuestados indican que todas las computadoras están conectadas a internet. Sin embargo, al valorar la conectividad, se observan los siguientes resultados.

Con respecto a las asignaturas en la plataforma CANVAS se da un hecho curioso: si bien las asignaturas utilizaban algún tipo de NTIC, tales como videoconferencias, diapositivas, programas televisivos, algunos servicios informáticos tales como multimedia, software, el uso de páginas web, entre otros, ninguno de estos servicios estaba disponible en la plataforma CANVAS para su uso a distancia. Esto cambió a partir del 9 de marzo del 2020, cuando se exigió que todas las asignaturas fueran subidas a esta plataforma, y así, actualmente el $100 \%$ de ellas está allí.

Figura 2.

Conectividad a internet de las computadoras del laboratorio de la UPAP filial Pilar.

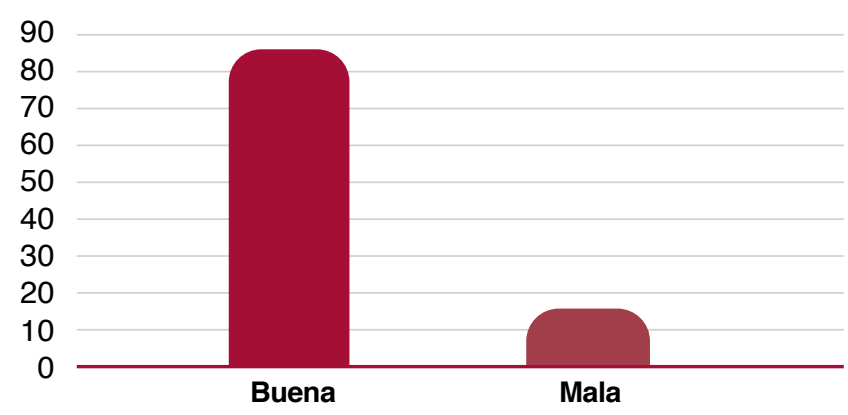

Fuente: Autores.

Obsérvese que, con respecto a la conectividad a internet, de un total de 6 computadoras que tiene el laboratorio de la UPAP, filial Pilar, el $84 \%$ tiene buena conectividad. Solo el $16 \%$ no la tiene, según las respuestas de los encuestados.

Figura 3.

Opinión de las clases digitales como una herramienta de las NTIC en la UPAP filial Pilar.

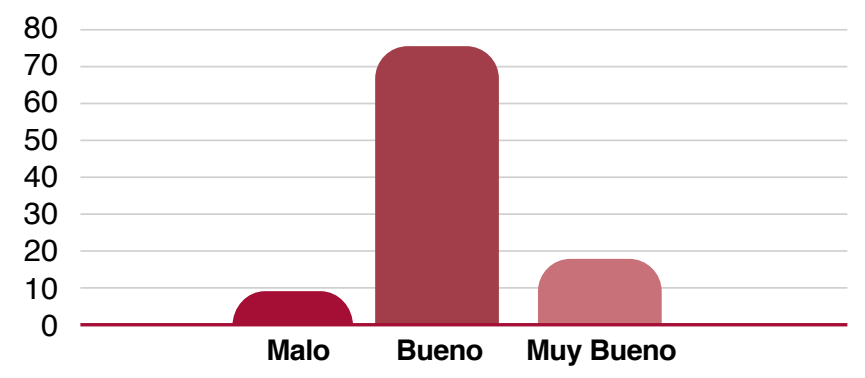

Fuente: Autores.

En este aspecto, con respecto a las clases digitales a través de la plataforma CANVAS, el $75 \%$ de los encuestados afirma que son buenas, el $17 \%$ que son muy buenas y solo un $8 \%$ dice que son malas. Esto indica que ha sido aceptable el uso de esta NTIC para darle continuidad al proceso enseñanza aprendizaje, lo cual favorecerá la continuidad del funcionamiento de la universidad.

NUEVAS TECNOLOGÍAS DE LA INFORMACIÓN Y LA COMUNICACIÓN, (NTIC): DIAGNÓSTICO EN LAS DIFERENTES CARRERAS QUE SE ESTUDIAN EN LA UPAP, FILIAL PILAR, PARAGUAY NEW INFORMATION AND COMMUNICATION TECHNOLOGIES (NTIC): DIAGNOSIS IN THE DIFFERENT CAREERS STUDIED AT UPAP, SUBSIDIARY PILAR, PARAGUAY 
Figura 4.

NTIC más utilizadas en la UPAP filial Pilar.

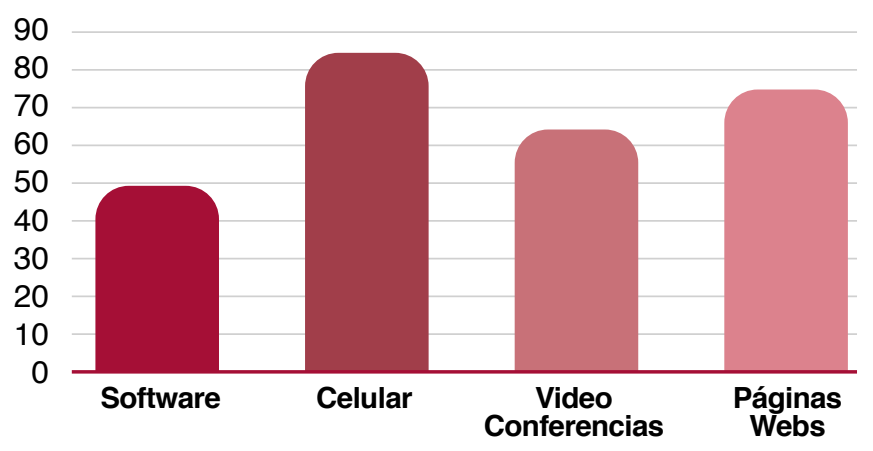

Fuente: Autores.

En este aspecto, se observa que el teléfono celular constituye la NTIC más utilizada por los estudiantes en las carreras (84\%). Se debe hacer, no obstante, una observación: esto no significa necesariamente que se use para fines estrictamente académicos, sino más bien para las redes sociales, que son otra herramienta de las NTIC. El $75 \%$ de los estudiantes usa las páginas web en busca de libros electrónicos, E-books, o materiales para trabajos de cada carrera. El $67 \%$ recurre a las videoconferencias y un $50 \%$ utiliza diferentes softwares, según la carrera, su perfil y sus necesidades.

Figura 5.

Uso de las NTIC en la UPAP filial Pilar.

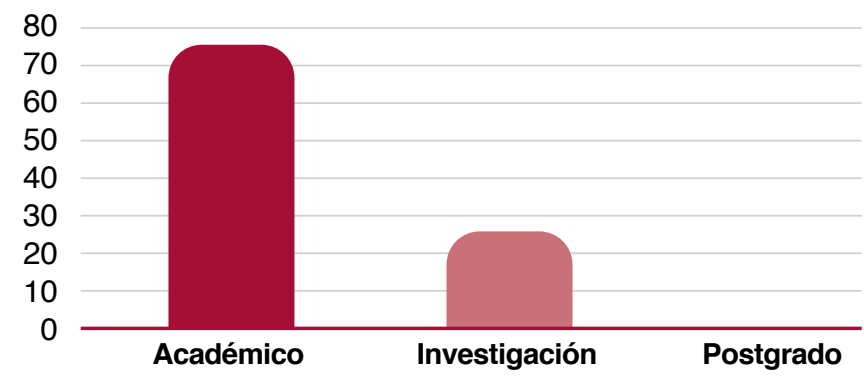

Fuente: Autores.

Este resultado es importante: las NTIC son mayoritariamente usadas en lo académico en un $75 \%$ de los casos y en un $25 \%$ para investigación. No se utilizan en el postgrado, lo cual indica que no existe postgrado, ni NTIC que permitan esto y en la investigación es poco significativo. Existe una observación: en la sede central de la UPAP, el uso de NTIC en postgrado se materializa en el curso de Aula Virtual para la Extensión Universitaria y en múltiples conferencias, lo cual no ocurre en la Filial de Pilar.

NUEVAS TECNOLOGÍAS DE LA INFORMACIÓN Y LA COMUNICACIÓN, (NTIC): DIAGNÓSTICO EN LAS DIFERENTES CARRERAS QUE SE ESTUDIAN EN LA UPAP, FILIAL PILAR, PARAGUAY NEW INFORMATION AND COMMUNICATION TECHNOLOGIES (NTIC): DIAGNOSIS IN THE DIFFERENT CAREERS STUDIED AT UPAP, SUBSIDIARY PILAR, PARAGUAY 
Figura 6.

Existencia de proyectos relacionados con las NTIC en las diferentes carreras en la UPAP filial Pilar.

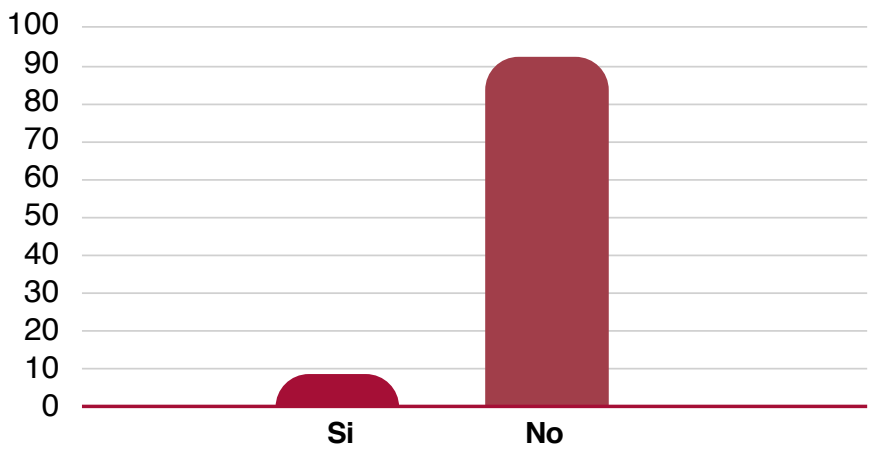

Fuente: Autores.

Al referirse a las principales competencias básicas y específicas de las NTIC que debería poseer un docente para lograr un buen desempeño, la mayoría de los encuestados coincidió en las siguientes:

- Gestión del conocimiento acerca del uso de las NTIC.

- Diseño de escenarios, plataformas y herramientas como NTIC.

- Reconocer la importancia y uso cada vez más necesario y eficiente de las NTIC.

En relación con los retos o desafíos que deben considerarse para lograr la efectividad de las NTIC en las carreras y universidad, se destacan:

- Mayor capacitación en NTIC.

- Ser más innovadores y autónomos para el desarrollo y gestión del conocimiento.

- Mayor conectividad y equipamiento.

Gran parte de los encuestados coincide en que las aplicaciones de las NTIC que deben potenciarse más son: medios audiovisuales, programas televisivos, servicios informáticos tales como multimedia, software, plataformas, páginas web y todas sus potencialidades, y las redes sociales.

En cuanto al uso que le dan hoy los estudiantes a la internet, salen a relucir, en orden de importancia, los siguientes:

Redes sociales

Juegos

Compras

Buscar trabajo

Estudios

Investigación

NUEVAS TECNOLOGÍAS DE LA INFORMACIÓN Y LA COMUNICACIÓN, (NTIC):

DIAGNÓSTICO EN LAS DIFERENTES CARRERAS QUE SE ESTUDIAN EN LA UPAP,

FILIAL PILAR, PARAGUAY

NEW INFORMATION AND COMMUNICATION TECHNOLOGIES (NTIC): DIAGNOSIS IN THE DIFFERENT CAREERS STUDIED AT UPAP, SUBSIDIARY PILAR, PARAGUAY 
Al evaluar productos elaborados y utilizados en las carreras como NTIC, solo se destacan los videos tutoriales y algunos materiales educativos digitales, lo cual es poco significativo.

En sentido general aparecen tres elementos que en opinión de todos los encuestados influyen en el uso de las NTIC en las carreras.

Conocimiento y manejo de las NTIC

Equipamiento

Conectividad a internet

Por último y no menos importante, se puede apreciar que hay dos carreras que no incorporan bibliografía que conozcan del uso de las NTIC, lo cual refuerza muchos de los ítems diagnosticados donde la falta de conocimiento es un elemento que resalta como aspecto a resolver.

Un $17 \%$ no incorpora ninguna fuente bibliográfica relacionada con las NTIC en sus carreras.

Figura 7.

Carreras que en su encuesta incorporan bibliografías que conocen y usan de las NTIC en la UPAP filial Pilar.

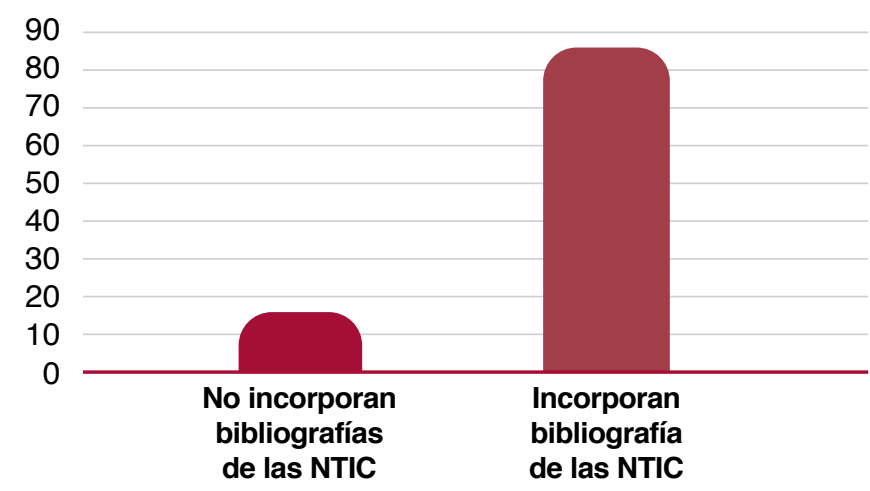

Fuente: Autores.

Se describen recomendaciones a considerar para introducir mejoras en el proceso enseñanza-aprendizaje en las carreras universitarias de la UPAP, derivadas de los resultados de la encuesta entrevista.

A partir de los resultados de la encuesta entrevista acerca del uso de las NTIC y su diagnóstico en las carreras de la UPAP, filial Pilar, salen a relucir las siguientes necesidades:

- Debe lograrse mayor cantidad de computadoras y equipamiento en la UPAP, filial Pilar, para el uso de las NTIC.

- Debe lograrse mayor conectividad y velocidad en la internet.

- Debe mejorarse la infraestructura en términos de laboratorios y aulas para clases virtuales y biblioteca para búsquedas virtuales de información.

- Deben potenciarse desde la UPAP filial Pilar y sus carreras, plataformas elaboradas por los docentes y estudiantes aprovechando los trabajos de tesis, para adecuar más las herramientas al contexto académico.

- Es importante propiciar una mayor capacitación docente acerca de las NTIC en general y en particular por carrera.

- Potenciar la investigación y el postgrado en las carreras de la UPAP filial Pilar, que posibilite el uso de las NTIC en sus programas.

- Elaborar proyectos de investigación con financiamiento, que permita acceder a los recursos necesarios por carrera para lograr un mayor uso y eficiencia de las NTIC.

NUEVAS TECNOLOGÍAS DE LA INFORMACIÓN Y LA COMUNICACIÓN, (NTIC):

DIAGNÓSTICO EN LAS DIFERENTES CARRERAS QUE SE ESTUDIAN EN LA UPAP,

FILIAL PILAR, PARAGUAY

NEW INFORMATION AND COMMUNICATION TECHNOLOGIES (NTIC): DIAGNOSIS IN THE DIFFERENT CAREERS STUDIED AT UPAP, SUBSIDIARY PILAR, PARAGUAY 


\section{Conclusiones}

Se realiza el diagnóstico del uso de las NTIC en las diferentes carreras habilitadas y que se estudian en la UPAP, filial Pilar, en Paraguay, como institución de la educación superior, en el contexto del proceso enseñanza - aprendizaje, en el año 2020 en momentos de la pandemia de COVID-19.

Este diagnóstico aportó las líneas de trabajo en función de buscar estrategias que permitan resolver los problemas encontrados, básicamente mayor gestión y capacidades de conocimiento de las NTIC y mayor equipamiento, conectividad y ancho de banda de internet, así como la elaboración de proyectos I+D hacia una potenciación del financiamiento, y plataformas y herramientas como NTIC para uso en las diferentes carreras.

Se proponen, en función de los resultados del diagnóstico de las NTIC en la UPAP filial Pilar, así como de forma general, recomendaciones sobre futuras estrategias pedagógicas para su implementación en las carreras que se estudian en esta institución de la educación superior, que puedan profundizarse y generalizarse como herramienta para construir el conocimiento.

Se entiende, a partir de los hallazgos de este trabajo, que una manera eficiente de desarrollar competencias en NTIC, podría ser un curso básico, ordenando los temas y actividades en torno a ejes temáticos, donde al mismo tiempo se puedan realizar operaciones y procesos que suponen actitudes, nuevas habilidades y conocimientos para todos, involucrando a todas las carreras.

El equipamiento y conectividad a internet debe permitir una mejor y más rápida navegación de banda ancha, preferentemente a través de wifi. Mejorar el mobiliario y aulas laboratorios para hacer más eficiente el uso de las NTIC. Se reconoce la ausencia de la investigación, el postgrado, los proyectos I+D en busca de resultados y de los recursos tecnológicos para el desarrollo de las NTIC.

El principal aporte concluyente del trabajo es fotografiar el escenario actual en relación con las NTIC en las carreras de la UPAP filial Pilar y orientar acciones para resolver o todo o parte del problema, dado que, a nivel nacional, existen todavía insuficientes trabajos en esta dirección.

Es importante resaltar que la principal limitación de esta investigación está en el diseño y el tiempo del trabajo, los resultados indican un problema y trazan un camino como punto de partida para hacer más estudios en relación con este tema de las NTIC. Los autores sugieren la continuidad de estudios en este tema para profundizar los análisis, y su extensión a otras filiales, como antecedente y punto de partida.

NUEVAS TECNOLOGÍAS DE LA INFORMACIÓN Y LA COMUNICACIÓN, (NTIC): DIAGNÓSTICO EN LAS DIFERENTES CARRERAS QUE SE ESTUDIAN EN LA UPAP, FILIAL PILAR, PARAGUAY NEW INFORMATION AND COMMUNICATION TECHNOLOGIES (NTIC): DIAGNOSIS IN THE DIFFERENT CAREERS STUDIED AT UPAP, SUBSIDIARY PILAR, PARAGUAY 


\section{Referencias Bibliográficas}

Álvarez, A. (2020). Informe final de las NTIC en las Carreras de la UPAP. Taller Metodológico. UPAP, Filial Pilar, 10 p.

ANEAES. (2013). Modelo Nacional de Acreditación de la Educación Superior. Recuperado el 19 de Agosto de 2016 de http://www.aneaes.gov.py/ aneaes/

ANEAES. (2016). Programas de Postgrado Acreditados. Recuperado el 16 de agosto de 2016 de http://www.aneaes.gov.py/aneaes/index.php/

Argüello, R. (2018). Efectos de las tecnologías educativas en el aprendizaje de la Historia de la Educación del Paraguay durante la posguerra de 1870, en estudiantes de la Facultad de Filosofía de la Universidad Nacional de Asunción. 2018. [Tesis de Doctorado, Facultad de Filosofía. Universidad Nacional de Asunción]. $64 \mathrm{p}$.

Ávila, E. (2003): Las Nuevas Tecnologías de la Información y la Comunicación como herramientas necesarias en la formación profesional de los estudiantes universitarios. Revista Ética.net. (1). 5 p. file:///C:/Users/user/Desktop/LIBROS\%20DIGITALES/Dialnet-LasNuevasTecnologiasDeLalnformacionYLaComunicacion-6871673.pdf

Badilla, M. G. (2010) Análisis y evaluación de un modelo socio constructivo de formación permanente del profesorado para la incorporación de las TIC. [Tesis doctorando, Universidad Ramón Liull]. 571 p. https://www.tdx.cat/bitstream/handle/10803/9246/Tesis_GracielaBadilla.pdf

Baptista, P. Hernández, R. y Fernández, C. (2014). Metodología de la Investigación (6a ed.). McGraw-Hill Interamericana.

Zárate, C. B., Carbajal Llanos, Y., Velasquez Muñoz, R, Figueredo Echeandí, C. y Robles Mori, H. (2017). Metodología de la Investigación, Manual para uso exclusivo de los estudiantes. Unidad Académica de Estudios Generales. Universidad de San Martín de Porres. 79 p. https://www.usmp.edu.pe/ estudiosgenerales/pdf/2017-I/MANUALES/METODOLOGIA\%20DE\%20LA\%20INVESTIGACION.pdf

CONACYT (s.f.) (https://www.conacyt.gov.py/)

Domínguez, Y. (2016). La utilización de las Tecnologías de la Información y las Comunicaciones (TIC) en el aprendizaje universitario. Revista Universidad y Sociedad, 8(4), 158-163. Recuperado de http://rus.ucf.edu.cu/

Universidad Politécnica y Artística de Paraguay UPAP (s.f.). (https://upap.edu.py/)

Valenzuela, J. (2017) TIC en la Profesionalización Docente: Necesidades Formativas y de Contexto Identificados en un curso de didáctica universitaria Tesis de Maestría en Investigación Científica. Facultad de Ciencias Jurídicas, Políticas y de la Comunicación. Universidad Autónoma de Asunción. 180 p.

\section{NUEVAS TECNOLOGÍAS DE LA INFORMACIÓN Y LA COMUNICACIÓN, (NTIC): DIAGNÓSTICO EN LAS DIFERENTES CARRERAS QUE SE ESTUDIAN EN LA UPAP, FILIAL PILAR, PARAGUAY NEW INFORMATION AND COMMUNICATION TECHNOLOGIES (NTIC): DIAGNOSIS IN THE DIFFERENT CAREERS STUDIED AT UPAP, SUBSIDIARY PILAR, PARAGUAY}

